\title{
Rol de enfermería en la consulta de prediálisis en el paciente con enfermedad renal crónica avanzada
}

\author{
Jenny Forero Villalobos, Silvia Barrios Araya \\ Pontificia Universidad Católica de Chile. Chile
}

\section{Resumen}

Introducción: El paciente con enfermedad renal crónica (ERC) tiene necesidades concretas de cuidado que conllevan al profesional de enfermería nefrológica a generar una atención integral e individualizada con el fin de detectar y disminuir riesgos a través de la consulta predialítica y educar a los profesionales del área de la salud acerca de su labor en dicha actividad.

Objetivo: Identificar y evaluar la efectividad de la consulta predialítica realizada por el profesional de enfermería en pacientes diagnosticados con enfermedad renal crónica, a través de la revisión sistemática de la literatura.

Metodología: Búsqueda electrónica en base de datos PUBMED, Meta buscadores como google académico, Epistemonikos, Scielo, Bireme, y CUIDEN. Se consideraron 47 artículos de los cuales 22 son cuantitativos y 25 cualitativos, en pacientes adultos de 18 a 80 años, entre los años 2005-2015.

Resultado: La consulta predialítica permitirá realizar intervenciones de diagnóstico temprano, educación y evaluación durante las primeras etapas de la ERC, reduciendo la morbi-mortalidad, retrasando la progresión de la enfermedad, por lo cual es necesario rediseñar los programas de gestión en enfermedades crónicas, enfatizando la colaboración de una red sanitaria liderada por el profesional de enfermería especialista que gestione los recursos existentes para el manejo de esta patología.

\section{Correspondencia:}

Jenny Forero V'illalobos

Doctor Ramón Rubio 282. El Ciprés Rengo/Rancagua- Chile E-mail: jjforero@uc.cl
Conclusión: La consulta predialitica ofrece beneficios tangibles al enfermo renal en las áreas de prevención de la ERC, adherencia a las recomendaciones del profesional de salud y la elección de la terapia.

\section{PALABRAS CLAVE}

- CONSULTA

- ENFERMERÍA

- PREDIÁLISIS

- PREVENCIÓN

- ELECCIÓN DE LA TERAPIA

Nursing role in predialysis visit in patients with advanced chronic kidney disease

\section{Abstract}

Introduction: Patients with chronic kidney disease have specific care needs that lead to professional nephrology nursing to generate a comprehensive and individualized attention in order to identify and reduce risks through predialysis consultation and educate other healthcare professionals about their work in the activity.

Objective: To identify and evaluate the effectiveness of predialysis consultation by nurses in patients diagnosed with chronic kidney disease, through the systematic review of the literature.

Methodology: Electronic search of PubMed database, Meta search engines like Google Scholar, Epistemonikos, Scielo, Bireme, and care. 47 articles, 22 were quantitative and 25 qualitative, in adults 18 to 80 and between 2005-2015 were considered. 
Result: The predialysis consultation would help to make early diagnosis interventions, education and evaluation during the firsts stages of CKD, reducing morbidity and mortality, slowing the disease progression, making it necessary to redesign programs in chronic disease management, emphasizing the collaboration of a network led by the health professional specialist nurses to manage existing resources to the management of this condition.

Conclusion: The query proves that predyalisis consultation provides tangible benefits to renal patients in the areas of prevention of CKD, adherence to the recommendations of the health professional and the choice of therapy.

\section{KEYWORDS}

- CONSULTING

- NURSING

- PRE-DIALYSIS

- PREVENTION

- CHOICE OF THERAPY

\section{Introducción}

La carga global de las enfermedades crónicas no transmisibles y entre ellas la enfermedad renal crónica, ha ido en aumento en el mundo, se estima que $10 \%$ de la población mundial tiene ERC y el $90 \%$ de las personas que la padecen no lo saben, esta población se duplicarán en los próximos 10 años ${ }^{1}$.

Alrededor de 1,5 millones de pacientes en el mundo están en diálisis o con trasplante renal, transformándose en un problema de salud pública debido al porcentaje de recursos económicos enfocados al tratamiento de las personas diagnosticadas con esta patología ${ }^{1}$. Por lo cual la OMS a promovido un plan de acción mundial enfocado a la prevención y control de las enfermedades no trasmisibles por lo cual es necesario implementar programas de prevención en todas las etapas de la enfermedad renal ${ }^{2,3}$.

Chile no está ajeno a esta realidad dado que la incidencia de ERC corresponde al $2,7 \%$ de la población en $>$ a 15 años en etapa III o más, lo que significa una pérdida de al menos el $50 \%$ de la función renal ${ }^{4,5}$. Actualmente existen 17.586 pacientes adultos con IRC en hemodiálisis crónica (HDC), cifra que aumenta, en 40 pacientes por millón de población al año4. Situación similar suce- de en la población chilena donde un $14,9 \%$ del gasto total del programa de las garantías explicitas de salud (GES) está destinada al tratamiento de esta ${ }^{6,7}$.

El paciente con ERC tiene un curso progresivo de su enfermedad en varios estadios, teniendo la necesidad de acceder a un tratamiento sustitutivo renal (TSR), haciéndolos acreedores de necesidades concretas de cuidado que permiten al profesional de salud especialista en el área de enfermería nefrológica generar una atención integral, individualizada, continua, con un enfoque interdisciplinario de tratamiento permitiendo el desarrollo de una comunicación terapéutica que asegure una buena y oportuna toma de decisiones compartidas por el paciente sus familiares y el profesional de enfermería ${ }^{8}$.

Fomentar conductas preventivas, educar a todos los profesionales acerca de su papel clave en la detección y reducción del riesgo de ERC, particularmente en poblaciones de alto riesgo deberá ser un papel crucial en el especialista de enfermería. Las publicaciones sobre la "consulta de enfermería a pacientes renales" en la fase de pre diálisis son escasas, por lo que los profesionales se han ido formando basados en la experiencia clínica obtenida a lo largo del tiempo?.

La situación antes descrita ofrece una oportunidad para otorgar a los profesionales de enfermería una visión amplia de la importancia y eficacia de su intervención a través de la consulta pre-dialítica en las áreas de prevención, elección, inducción de la terapia, teniendo en cuenta que cualquier cambio de modalidad de TSR debe ir precedida de un proceso de elección informada por parte del paciente, generando igualmente recomendaciones adaptadas al contexto en las intervenciones del profesional de enfermería en el cuidado de pacientes con ERC.

Revisión integrativa cuya metodología utilizada fue la búsqueda electrónica en las bases de datos PUBMED, meta buscadores como Google académico, EPISTEMONIKOS, SCIELO, BIREME, CUIDEN con interfaz de búsqueda de términos Mesh y Clinical Queries. Se consideraron estudios cuantitativos, cualitativos realizados en pacientes adultos de 18 a 80 años, entre los años 2005-2015, sin restricción de idioma. Las palabras clave utilizadas fueron: consulta, ERC, prevención, adherencia, elección de la terapia, unidos dichos términos por los operadores boléanos "and" y "or"; los criterios de inclusión definidos fueron: artículos teniendo como población objetivo personas diagnosticadas con ERC etapas 3-5 entre (18 a 80 
años), artículos donde se analice el desarrollo de la consulta predialitica. Los criterios de exclusión fueron: Estudios publicados antes del 2005, artículos que solo contemplen pacientes en programas de remplazo renal en un periodo mínimo de 3-6 meses.

Se encontraron 46 artículos, 22 en inglés y 24 en español, publicados entre 2005 y 2015 que cumplían los criterios de inclusión descritos anteriormente; se procedió a la lectura crítica cuidadosa realizando análisis completo a través de fichas bibliográficas y valoración general de la calidad metodológica de cada uno de los estudios incluidos de acuerdo a cada tipo de diseño metodológico.

\section{Consulta predialítica}

En la actualidad el enfoque sanitario que lidera la atención primaria sigue siendo el modelo biomédico tradicional, con el que otorgar atención a personas con enfermedades crónicas, específicamente ERC. EI médico general, es el primer profesional que tiene contacto con este tipo de pacientes haciendo la función de puente comunicador con otros profesionales de la salud ${ }^{10}$.

Es importante destacar que para esta atención el profesional médico cuenta con tiempos reducidos, dada las exigencias en relación a las metas de cobertura planificadas en atención primaria. A lo anterior se suma la comunicación limitada con especialistas y con otros profesionales formados en el área de la nefrología, la falta de infraestructura y déficit de presupuesto destinado para la reducción de los factores que aumentan la prevalencia de esta enfermedad ${ }^{11}$.

Al respecto la literatura señala que es necesario rediseñar los programas de gestión en enfermedades crónicas, enfatizando la colaboración de una red sanitaria que permita optimizar los recursos existentes, disminuyendo la demanda requeridas por los pacientes con ERC sobre el sistema de salud ${ }^{12}$.

Diferentes autores plantean que este trabajo en red debiese centrarse principalmente en factores para retrasar o prevenir la progresión de la ERC con el fin de disminuir la incidencia de pacientes que requieren alguna terapia de reemplazo renal, promoviendo estilos de vida saludable que le permitan al paciente el autocuidado de su propia salud y automanejo de su enfermedad ${ }^{13,14,15}$.

Dentro de las propuestas planteadas para mejorar la atención del paciente con ERC diversos estudios reportan los beneficios de la atención por parte de un equipo multidisciplinar el cual abarca de manera positiva todas las intervenciones preventivas tempranas durante las primeras etapas de la ERC a partir de un protocolo que tiene cuatro componentes principales: la educación del paciente, gestión administrativa, asesoramiento dietético y evaluación del cumplimiento de los objetivos clínicos ${ }^{16,17,18}$.

En contraste con el modelo biomédico tradicional, la atención multidisciplinar está configurada para individualizar el cuidado necesario cubriendo las áreas de mayor demanda, con el objeto de lograr un estado superior de bienestar o calidad de vida para el paciente $^{19}$. El enfoque multidisciplinar en el caso de la $E R C$, requiere de un equipo de salud especializado en cuidado renal y propone 3 alternativas de abordaje: la consulta pre-dialítica, el protocolo reno-protector y el cuidado integral del paciente renal, donde los pacientes en estadios tempranos especialmente 3-4, consultan de forma programada ${ }^{9,20,21}$.

Las diferentes alternativas de abordaje, le permiten por un lado, al paciente acceder de forma exclusiva a consultas que le permitan resolver conflictos decisionales, a través de la aplicación de un proceso de toma de decisiones conjunta destinado a proporcionar una información completa y equilibrada sobre las distintas modalidades de terapia de sustitución renal, donde el paciente sea el que decida finalmente respecto a sus preferencias ${ }^{22}$. Informando anticipadamente al paciente y su familia con la estandarización y protocolización, seguimiento la implantación con suficiente antelación de un acceso (AV o catéter) idóneo; la vacunación de Hepatitis $B^{23}$.

Los beneficios de la consulta pre-dialítica encontrados fueron: intervenciones de diagnóstico temprano, educación y evaluación durante las primeras etapas de la ERC que reducen la morbilidad y la mortalidad, retrasando la progresión de la enfermedad renal ${ }^{16,24}$. Además lo analizado según la literatura, los pacientes que son captados de forma tardía tienen indicadores de daño renal deficientes comparados con los captados de forma temprana ${ }^{25,26}$.

Es decir, trabajar bajo esta propuesta influirá directamente en la calidad de vida y el tiempo de ingreso a diálisis, los tiempos involucrados sobre la probabilidad de sobrevida libre de diálisis la literatura reporta a 12 meses aproximadamente un $87 \%$. Luego de dos años de seguimiento, esta probabilidad fue de $76 \%$. 
El tiempo promedio del total de pacientes sin requerir de diálisis fue de 50.41 meses (IC 95\% 40.77, 60.04). Resaltando el ahorro directo por parte del sistema de salud por cada mes que se retarde el ingreso a diálisis de un paciente ${ }^{20,26}$. Permitiendo programar el ingreso a la terapia de sustitución renal evitando situaciones de urgencia a través de la planificación de los parámetros de autocuidado esperados, lo que se traduce en valores bioquímicos positivos, proporcionando más seguridad al paciente para afrontar el manejo de su enfermedad ${ }^{20}$.

Otro componente importante de la consulta pre-dialítica es la educación, se ha demostrado que el aumento de conocimientos por parte del paciente sobre los distintos aspectos de la enfermedad (causas, tratamiento, dieta...) disminuye el temor y la ansiedad, aumentando las expectativas del tratamiento ${ }^{27}$.

Autores reportan que informar sobre los factores como elección e inducción a la terapia, actividades de prevención que fomentan el autocuidado trabajando en la prevención de factores de riesgo, disminuyen las tasas de hospitalización en aproximadamente en un $17 \%$, generando una mayor duración de la terapia de diálisis, en comparación a la atención estándar ${ }^{25,27 .}$

\section{Focos de la consulta predialítica}

\section{A.Prevención de la ERC}

Dentro de las herramientas claves en la promoción y prevención primaria de la ERC es necesario:

a) Incentivar hábitos de vida saludable en cuanto a alimentación adecuada, actividad física, restricción de elementos nocivos (alcohol, hábito tabáquico, consumo de drogas), evitar uso de medicamentos nefrotóxicos tales como AINES, medios de contraste, corticoides, entre otros.

b) Identificar pacientes en riesgo a través de la historia médica y quirúrgica considerando: morbilidades como la diabetes, hipertensión y/o enfermedad cardiovascular haciendo hincapié en el control adecuado de las mismas; historia de enfermedad renal en familiares de primer grado, considerando en forma especial, aquellas con componente genético o hereditario; historia personal nefrourológica (infección urinaria alta recurrente, alteraciones anatómicas, cálculos urinarios); así como también factores dietarios, sociales, demográficos y culturales. c) Igualmente debe considerarse el diagnóstico temprano de la ERC con el fin de limitar la extensión de la misma y el desarrollo de complicaciones teniendo en cuenta la clasificación de los estadios a través de la velocidad de filtración glomerular VFG, desde el valor de la creatinina sérica, la presencia de proteinuria y albuminuria facilitando su pesquisa y manejo oportuno $19,29,30$.

Como prevención secundaria, en el programa cardiovascular del MINSAL se propone que el manejo terapéutico en el estadio III apunta al control de la presión arterial, especialmente mediante el bloqueo del sistema renina-angiotensina- aldosterona a través del uso de medicamentos como agentes de protección cardio-renal (IECA/ARA II) como se ha descrito hasta en un $70 \%$ de la población en consulta predialítica y el uso del ácido acetilsalicílico, estatinas y vitamina $D^{13,24,30}$.

En la consulta predialítica el profesional de enfermería especialista tiene un rol fundamental en la educación para la salud donde es necesario instruir respecto a la presentación clínica de la uremia y los factores de riesgo desencadenantes, las complicaciones asociadas con la progresión renal y una introducción a las diversas terapias de sustitución renal (TRR); Hemodiálisis (HD), Diálisis Peritoneal (DP), Trasplante renal (TR); y tratamiento conservador ${ }^{31}$.

En ambos niveles de prevención, estudios de intervención individuales liderados por el profesional de enfermería muestran el beneficio de la modificación en los estilos de vida incentivando los hábitos saludables, orientación nutricional, restricción de sodio en la dieta, regulación de los lípidos, control glucémico, la albuminuria, control en el uso de sustancias y medicamentos nefrotóxicos, entre otros, aún en pacientes que ya se encuentran con diagnóstico de ERC ${ }^{16,18}$.

En estadíos más avanzados el cuidado se centra en el manejo de las complicaciones asociadas con la ERC y la TRR, dichas medidas como parte de la prevención terciaria consideran, la evaluación y cuidado del acceso vascular (catéter o fístula arteriovenosa) o peritoneal; el esquema de vacunación de hepatitis $B$; el seguimiento adecuado de la mineralización ósea y metabolismo de calcio y fósforo; tratamiento de infecciones asociadas a la atención en salud; el registro para su inclusión en la lista de espera para trasplante renal y la corrección de eventos potencialmente mortales, como la hiperpotasemia, insuficiencia cardiaca y acidosis metabólica entre otras $22,24,31,33,34$. 


\section{B. Adherencia a las indicaciones del equipo de salud}

Otro foco central a considerar en la consulta predialítica es fomento de la adherencia a las diferentes indicaciones del equipo de salud en los diversos niveles de atención en salud.

Lo anterior con el objetivo de evitar o atenuar la progresión de la enfermedad renal y el ingreso a TRR y una vez aquellos que están en alguna terapia mejorando la calidad de vida del paciente.

Sumado a lo anterior algunos estudios indican que la necesidad del paciente de someterse a un estricto, invasivo y continuo tratamiento; el cual implica cambios importantes en su estilo y hábitos de vida, cambios a nivel social (restricción en su tiempo disponible), cultural (cambios en su alimentación) y emocional (impacto negativo por intervenciones dolorosas) son condiciones que podrían modificar la adherencia con el detrimento de la salud del paciente ${ }^{28,35}$.

En los países desarrollados, las tasas de adherencia a los tratamientos en enfermedades crónicas se sitúan alrededor del $50 \%$, cifra considerablemente menor en los países en vías de desarrollo, según un reciente informe de la Organización Mundial de la Salud (OMS), que califica la falta de adherencia como un «problema mundial de gran magnitud»36.

Considerando la adherencia como parte vital, no solo de la adecuada terapia para el paciente, sino del empoderamiento del mismo hacia su tratamiento se hace vital detectar factores que influyan en la misma, como dificultad para la toma de medicamentos, alimentación e ingesta de líquidos restrictivas, cumplimiento de horarios en forma estricta, entre otros; añadiendo que las complicaciones derivadas de un tratamiento incompleto o administrado de forma incorrecta aumentan los costos asistenciales con la sobrecarga en el sistema de salud y desenlaces desfavorables hacia el paciente ${ }^{29}$.

\section{Adherencia farmacológica y enfermedades crónicas}

La adherencia terapéutica es un fenómeno complejo condicionado por múltiples factores de diversa naturale$\mathrm{za}$, sin embargo, es un comportamiento humano, modulado por componentes subjetivos y en esta dimensión debe ser esencialmente comprendido, abordado e investigado, en general, el $20-50 \%$ de los pacientes no toman sus medicamentos, aunque la tasa de incumplimiento puede variar mucho según la patología ${ }^{37}$.
En el contexto de las enfermedades crónicas, la OMS considera la falta de adherencia un tema prioritario de salud pública debido a sus consecuencias negativas: fracasos terapéuticos, mayores tasas de hospitalización y aumento de los costos sanitarios ${ }^{38}$.

En la actualidad la mayoría de los pacientes que precisan tratamiento sustitutivo de la función renal presentan comorbilidades asociadas (Enfermedad cardiovascular, diabetes mellitus, ateromatosis, hipertensión arterial, etc.) siendo necesario ser tratados con un elevado número de fármacos, aumentando el riesgo de abandono al tratamiento, haciendo más difícil el alcanzar los objetivos terapéuticos como el control de la presión arterial, glucémico, el metabolismo óseo y hematopoyético ${ }^{38}$.

La falta de adherencia farmacológica incluye aspectos como la omisión de tomas, reducción de la dosis prescrita, intervalos o frecuencias de administración incorrectos, sumado al no ajuste a los requerimientos de administración en relación con las comidas u otras circunstancias. Algunas como consecuencia de actos involuntarios (olvidos o confusión) considerando horarios de administración diferentes a los tradicionalmente establecidos, esquemas de dosis complejos o conocimiento limitado respecto a la indicación de los fármacos, fenómenos documentados hasta en un $20 \%$ de los pacientes, bien sea en DP o en HD ${ }^{38,40}$.

\section{Adherencia a restricción de fluidos}

Una de las funciones más importantes del tratamiento con diálisis es corregir el desequilibrio hídrico, conseguir un óptimo estado de hidratación, sin fluctuaciones y con mayor estabilidad hemodinámica.

La sobre hidratación se produce por una ingesta de líquidos superior a las pérdidas lo que indica que es la ultrafiltración y la diuresis residual es menor a la ingesta de líquidos. Lo que conlleva a presentar edemas, elevación de la presión arterial, aumento de peso y en casos graves insuficiencia cardíaca. En su diagnóstico debe descartarse el déficit de ultrafiltración, su profilaxis radica en el control de la ingesta de líquidos, la dieta hiposódica y el uso adecuado de las concentraciones sodio ${ }^{40}$.

\section{Adherencia a las indicaciones nutricionales}

La falta de adherencia a la nutrición se ha documentado en diferentes estudios, evidenciando que los pacientes tenían más dificultades con las recomendaciones dieta- 
rías, el $16 \%$ fueron clasificados como no adherentes y el $26 \%$ admitieron alterar las indicaciones nutricionales recomendadas por el profesional de la salud ${ }^{42,43}$. Las costumbres sociales (comida en familia, alimentación disponible según zona geográfica, tipo de alimentación según la cultura) representan otra barrera que dificulta la adherencia a las restricciones dietarias ${ }^{42}$.

Por lo anterior, como herramienta para aumentar la adherencia en los diferentes niveles, es necesaria la existencia de una comunicación eficaz y satisfactoria para proporcionar información necesaria de modo que favorezca y garantice niveles mínimos de comprensión por parte del paciente y para esto es importante que durante la consulta predialitica el profesional de enfermería sea flexible adaptándose a las situaciones y características individuales de los pacientes, identificando los elementos que afectan el comportamiento del mismo ${ }^{29,35}$.

\section{Elección de la terapia}

Este foco corresponde al proceso de información y de elección de la modalidad de diálisis en pacientes IRC constituye un derecho del paciente y una etapa clave para el tratamiento de la persona que padece dicha enfermedad ${ }^{44}$. Un estudio analizado respecto a la toma de decisión respecto a la elección de la terapia indica que aproximadamente en un $17,5 \%$ de las ocasiones fue tomada por el médico, por el paciente en un $71 \%$ y en un $11 \%$ conjuntamente entre médico y paciente ${ }^{23}$.

Por otra parte el $12,2 \%$ de los pacientes no decidieron, ya que ellos creyeron que esta patología era reversible y no necesitarían algún tipo de terapia para mejorar su calidad de vida con el paso de los años, lo anterior relacionado altas tasas de filtración glomerular en la valoración inicial de paciente ${ }^{23}$.

Por tal motivo el papel del profesional de enfermería en la consulta pre-dialítica es fundamental, garantizando que este proceso de selección de la terapia sea realizado de manera integral, buscando la participación activa del paciente, la autonomía en el proceso de toma de decisión y la independencia en la elección de la terapia lo que podría asegurar un mayor compromiso del paciente con su auto-cuidado ${ }^{33}$.

Lo anterior permite al profesional de enfermería identificar las barreras como: la falta y comprensión de la información (literacidad), entendimiento del proceso dialítico y de las pautas de autocuidado que se deben adquirir según la modalidad, así como la preocupación por el aislamiento social y familiar en que se encuentra el paciente con el propósito de mejorar la atención y enfocar los recursos necesarios ${ }^{22,45}$.

Un estudio que a través la consulta predialítica en un periodo de 8 a 16 meses, analiza la elección de la terapia con diferentes factores como: la edad, sexo, nivel cultural, actividad laboral, etiología base, muestra que cuando el inicio a la terapia es en forma aguda de un $33 \%$, donde el $44 \%$ eligieron hemodiálisis, el $15 \%$ diálisis peritoneal, el $8 \%$ elige tratamiento sustitutivo conservador asistido en domicilio y porcentajes muy bajos de trasplantes en aproximadamente un 0,9\% ${ }^{22,23,46}$.

Igualmente se destaca como una de las ventajas del desarrollo de la consulta pre dialítica en el inicio programado de la hemodiálisis donde aproximadamente un $92 \%$ de los pacientes procedentes de la consulta disponía de acceso vascular permanente y funcional ${ }^{23,33}$.

Por otra parte los estudios publicados hasta la fecha demuestran que la DP tiene un coste económico significativamente inferior a la HD. Esta ventaja se optimiza si se usa la DP como modalidad de inicio, al permitir realizar diálisis a dosis bajas durante períodos más prolongados. En el momento de iniciar CAPD, la mayoría de pacientes vistos en la consulta disponían de catéter peritoneal funcional y todos iniciaron la técnica de forma programada en su domicilio $23,33,47,48$.

En general la educación sobre los tipos de modalidades existentes se asocia con un aumento en la elección de DP dado que se obtienen mejores resultados, menos riesgos, mayor satisfacción y menor costo, pese a esto la HD sigue siendo el tratamiento más elegido ${ }^{46}$.

Por tanto, la consulta pre-dialítica se convierte en un factor relevante al momento de seleccionar la terapia, sobre todo en la elección de DP, debido a que los pacientes que no han recibido información sobre esta modalidad no la eligen por miedo y/o desconocimiento ${ }^{49,50}$.

\section{Rol de la enfermera en la consulta predialítica}

Estudios analizados en la presente revisión reportan que uno de los elementos clave en la gestión de la ERC en pacientes con estadio 3 y 4 incluyen al enfermera/o con formación de especialista en el área de enfermería nefrológica, que desarrolle su rol profesional en las áreas asistencial, educativa, de gestión e investigación, en la consulta pre-dialítica y además que cuente con asesoría directa de un nefrólogo a través de diferentes modali- 
dades: contacto directo, telemedicina, entre otros, con posibilidad de derivación a otros profesionales de equipo de salud, de tal manera de formar protocolos acordes al trabajo de un equipo multidisciplinar, ya que los datos indican que la atención clínica para la creciente población de pacientes con ERC no puede depender exclusivamente de nefrólogos $11,14,22,24$.

Por consiguiente, la evidencia revisada apoya la intervención de enfermería especializada para mejorar la atención nefrológica ${ }^{17}$; dado que actúa como un administrador de casos, llevando a cabo una evaluación sistemática de los pacientes, sus problemas de salud, estado físico, psicológico y social, proponiendo actividades de cuidado dentro de un modelo organizado y fortalecido a través de terapias basadas en la evidencia, el apoyo a la autogestión, la comunicación entre los proveedores de salud y el examen de las tendencias determinando si los pacientes cumplen con los objetivos de tratamiento $0^{9,14,41}$.

Asimismo, el rol educador del profesional de enfermería es significativo, ya que cuenta con las competencias impartidas durante su formación para focalizar la educación y comprender la conducta del ser humano, lo que hace a este profesional altamente competente para realizar promoción y educación para la salud de la población a través de la potenciación del autocuidado ${ }^{11,51}$.

Por lo tanto, en la consulta pre-dialítica con programas de autocuidado personalizados, enfocados a las necesidades de cada paciente, junto al seguimiento periódico por enfermera/o, permiten identificar aspectos donde sea necesaria la reeducación, dándole a la persona herramientas para empoderarse en su automanejo y recursos que podrían utilizar, lo que disminuye la ansiedad derivada de la falta de información y de conocimientos, así como los reingresos derivados por el déficit de autocuidado en sus distintas áreas ${ }^{15,51}$.

Por consiguiente, en la consulta predialitica la valoración y entrevista del paciente en función de la etapa 0 estadio de la ERC en que se encuentra, es un determinante fundamental para definir el foco de la educación y atención a otorgar por el profesional de enfermería, y por ende el éxito de enlentecer la progresión de la enfermedad renal y así disminuir la incidencia de pacientes en diálisis.

Asimismo, dentro de la búsqueda se encontraron modelos de consulta realizada por profesional de enfermería indicando resultados positivos como se evidencia en el estudio CANPREVENT, el cual muestra que el cuidado médico apoyado por especialista de enfermería era costo-efectivo; dado que el paciente era visto inicialmente por enfermera/o y aquellos casos que requerían consulta por especialista, esta los derivaba al nefrólogo, lo que se traduce en menores costos ${ }^{19,24}$.

Otra ventaja observada, la da a conocer el ensayo MASTERPLAN, donde se compara la atención del paciente con ERC dada por profesionales de enfermería versus personal médicos, donde se establecía que el tiempo de atención directa brindado por el nefrólogo tenia un promedio de 12 minutos en comparación a la atención de enfermería, la cual no solo abordaba el tiempo de la consulta si no que incorporaba el tiempo de seguimiento continuo al paciente a través de medios telefónicos y/o correo electrónico, lo que sumado daba un tiempo de 187 minutos, obteniendo grandes índices de satisfacción con la atención del especialista de enfermería ${ }^{17,22,24}$.

Un aspecto de gran importancia destacado por el paciente durante la consulta era la actitud y el compromiso positivo del personal de enfermería, en términos de amabilidad y buena disposición para responder preguntas, permitiendo la participación de los miembros de la familia en el curso de la enfermedad ${ }^{16,52,53}$.

\section{Conclusiones}

La CP demostró ser eficaz para retrasar la progresión de la enfermedad renal crónica en los adultos que están en la fase de pre-diálisis por lo que es necesario implementar y evaluar intervenciones educativas enfocadas a los pacientes con etapas tempranas de la enfermedad renal crónica.

La enfermera al ser partícipe de la CP cumple un papel de gran importancia planeando, implementando y evaluando actividades de cuidado acordes al programa de educación y formación progresiva en: Información sobre la ERC y sus posibilidades de tratamiento para una elección independiente, generación de autocuidados y preparación para inicio de TRR.

Las consultas lideradas por el profesional de enfermería para el cuidado de la ERC son extremadamente útiles ya que dedican a este paciente el tiempo necesario para dar seguimiento a las intervenciones educativas cerciorándose, que entienda y asimile los conocimientos necesarios para cuidarse mejor.

Desde el concepto de prevención, es útil realizar actividades de cuidado enfocadas en el bienestar y satisfac- 
ción del paciente, mejorando las condiciones de vida y la percepción que estos tienen sobre su salud.

La falta de adherencia es importante dado que conlleva muchos resultados indeseables tales como: disminución de los beneficios terapéuticos, necesidad de mayores pruebas de diagnóstico y servicios médicos, así como una mayor necesidad de medicación.

El proceso de selección de la terapia deberá ser realizado de manera integral, buscando la participación activa del paciente, la autonomía en el proceso de toma de decisión y la independencia en la elección, lo que podría asegurar un mayor compromiso del paciente con su tratamiento.

Recibido: 2 enero 2016

Revisado: 20 enero 2016

Modificado: 22 enero 2016

Aceptado: 25 enero 2016

\section{Bibliografía}

1. World Kidney Day 2011. [Consultado el 2 de septiembre 2015].Disponible en: http://www. worldkidneyday.org/page/press-material

2. Comunicado de prensa OMS defunciones prematuras por enfermedades no trasmisibles Consultado: 2 de septiembre de 2015. Disponible en:http://www.who.int/mediacentre/news/ releases/2015/noncommunicablediseases/es/

3. Organización Panamericana de la Salud. Estrategia regional y plan de acción para un enfoque integrado sobre la prevención y el control de las enfermedades crónicas. OPS. Washington, D.C.: 2007

4. Encuesta nacional de salud. Chile 20092010.MINSAL/Departamento de Salud Pública PUC. Consultado:2de septiembre de 2015.http://web.minsal.cl/portal/url/item/ bcb03d7bc28b64dfe040010165012d23.pdf

5. Flores, J. C., Alvo, M., Borja, H., Morales, J., Vega, J., Zúñiga, C., ... \& Münzenmayer, J. (2009). Enfermedad renal crónica: Clasificación, identificación, manejo y complicaciones. Revista médica de Chile, 137(1), 137-177.

6. Ministerio de Salud. Guía Clínica Insuficiencia Renal Crónica Terminal. 1st Ed. Santiago: Minsal, 2005.

7. Poblete H. Sociedad Chilena de Nefrología Registro de diálisis. XXXI Cuenta de Hemodiálisis Crónica (HDC) en Chile [Internet]; 2012. [citado septiembre 2015]. Disponible en: http://www. asodi.cl.

8. Cepeda Pérez I, Viera Cordero DM, Ortega Ladrón de Cegama MP, Miguel Brea A, Lorenzo Méndez M, Sánchez Salán B. Diagnósticos de enfermería en la consulta de enfermedad renal crónica avanzada: continuidad de cuidados. Enfermería Nefrológica. 2012; 15:159-160.

9. Bardón-Otero E, Marti i Monros A. Enfermería en la consulta de enfermedad renal crónica avanzada (ERCA) Nefrología. 2008; Supl. 3, 53-56.

10. Kidney Health Australia. Chronic Kidney Disease (CKD) Management in General Practice. Melbourne: Kidney Health Australia, 2010.

11. Bonilla León FJ. Educación sanitaria al paciente con enfermedad renal crónica avanzada: ¿existe evidencia de su utilidad? Enfermería Nefrológica 2014;17(2):120-131.

12. Howard H, Salked G, White $S$ et al. The Cost Effectiveness of Early Detection and Intervention to Prevent the Progression of Kidney Disease in Australia. Melbourne: Kidney Health Australia, 2006.

13. Wong FKY, Chow SKY, Chan TMF. Evaluation of a nurse-led disease management programme for chronic kidney disease: a randomized controlled trial. Int J Nurs Stud 2010;47(3):268-278.

14. Barrett BJ, Garg AX, Goeree R, Levin A, Molzahn $A$, Rigatto $C$, et al. A nurse-coordinated model of care versus usual care for stage 3/4 chronic kidney diseases in the community: a randomized controlled trial. Clin J Am Soc Nephrol 2011 Jun;6(6):1241-1247.

15. Choi ES, Lee J. Effects of a face-to-face selfmanagement program on knowledge, selfcare practice and kidney function in patients 
with chronic kidney disease before the renal replacement therapy. Journal of Korean Academy of Nursing 2012;42(7):1070-1078.

16. Richards N, Harris K, Whitfield M et al. Primary carebased disease management of chronic kidney disease (CKD), based on estimated glomerular filtration rate (eGFR) reporting, improves patient outcomes. Nephrol Dial Transplant 2008; 23: 549- 55.

17. Van Zuilen $A$, Blankestijn $P$, Van Buren $M$, ten Dam M, Kaasjager K, Ligtenberg G, et al. Nurse practitioners improve quality of care in chronic kidney disease: two-year results of a randomised study. Neth J Med 2011;69:517-526.

18. Sciamanna CN, Alvarez $K$, Miller J, Gary T, Bowen M. Attitudes toward nurse practitionerled chronic disease management to improve outpatient quality of care. Am J Med Qual 2006; 21: 375-81.

19. Peeters $M J$, van Zuilen $A D$, van den Brand $J A$, Bots $M L$, van Buren $M$, Ten Dam MA, et al. Nurse practitioner care improves renal outcome in patients with CKD. J Am Soc Nephrol 2014 Feb;25(2):390-398.

20. Pabón RER, Nieto $M$, Corrado $P$, Martínez $A$, Escobar CM, Jaramillo C. Retardo en la progresión del daño renal en pacientes con Insuficiencia Renal Crónica estado 4, impacto de un programa de prevención en prediálisis. Revista Colombiana de Nefrología 2007;1(1):10-21.

21. Klebe $B$, Farmer $C$, Cooley $R$, de Lusignan $S$, Middleton R, 0'Donoghue D, New J, Stevens P: Kidney disease management in UK primary care: Guidelines, incentives and information technology. Family Practice 24: 330-335, 2007.

22. Selgas R, Aguilar J, Julián JC, Toledo A. Realidad y futuro en el tratamiento de la enfermedad renal crónica avanzada (ERCA). Nefrología 2007;27(6):689-69.

23. Álvarez Pérez R, Velasco Ballestero S. La consulta de predialisis de enfermería: logros y oportunidades de mejora. Revista de la Sociedad Española de Enfermería Nefrológica 2007;10(3):6-11.

24. Mason J, Khunti K, Stone M, Farooqi A, Carr S. Educational interventions in kidney disease care: a systematic review of randomized trials. American Journal of Kidney Diseases 2008;51(6):933-951.

25. Wu IW, Wang SY, Hsu KH, Lee CC, Sun CY, Tsai $\mathrm{CJ}$, et al. Multidisciplinary predialysis education de- creases the incidence of dialysis and reduces mor- tality - a controlled cohort study based on NFK/ DOQI guidelines. Nephrol Dial Transplant. 2009; 24: 3426-3433.

26. Strand H, Parker D. Effects of multidisciplinary models of care for adult predialysis patients with chronic kidney disease: a systematic review. International Journal of Evidence Based Healthcare 2012;10(1):53-59.

27. Pulido $F$, Arribas $E$, Pulido JF, González $F$, Aragonzillo I. Tres años en la consulta ERCA. Enferm Nefrol. 2009; 34: 250-251.

28. Contreras F, Esguerra G, Espinosa JC, Gutiérrez C, Fajardo L. Calidad de vida y adhesión al tratamiento en pacientes con insuficiencia renal crónica en tratamiento de hemodiálisis. Universitas Psychologica 2006;5(3):487-500.

29. Guerra Guerrero VT, Díaz Mujica $A E$, Vidal Albornoz K. La educación como estrategia para mejorar la adherencia de los pacientes en terapia dialítica. Revista Cubana de Enfermería 2010;26(2):52-62.

30. Alcázar R, De Francisco A. Acción estratégica de la Sociedad Española de Nefrología frente a la enfermedad renal crónica. Nefrología 2006;26(1):1-4.

31. Guías S. Factores de progresión de la enfermedad renal crónica. Prevención secundaria. Nefrología 2008(Supl 3):17-21.

32. Trujano T. Proceso de atención enfermero a persona con Insuficiencia renal crónica. Enf Neurol (Mex 2012;11(1):30-33.

33. García Estévez S, Vinagre Rea G, Arribas Cobo P. Influencia de factores epidemiológicos en la elección de la modalidad de tratamiento renal sustitutivo en la consulta de enfermería de enfermedad renal crónica avanzada. Enfermería Nefrológica 2012;15(4):291-295.

34. Orozco BR. Prevención y tratamiento de la enfermedad renal crónica (ERC). Revista Médica Clínica Las Condes 2010;21(5):779-789. 
35. Herrera Lian A. Factores que influyen en la adherencia a tratamientos en pacientes con riesgo de enfermedad cardiovascular. Avances en Enfermería 2008; Vol.26, núm.1; 36-42.

36. Adherence to long-term therapies. Evidence for action. World Health Organization 2003.

37. Cazorla Santana María Nieves, Rodríguez Díaz Dora. Grado de adherencia terapéutica a los fármacos del metabolismo óseo-mineral: ¿toman nuestros pacientes la medicación prescrita?. Enferm Nefrol [revista en la Internet]. 2013 Mar [citado 2015 Sep 21]; 16(1): 41-47. Disponible en: $\quad h t t p: / / s c i e l o . i s c i i i . e s / s c i e l o . p h p ? s c r i p t=s c i$ arttext\&pid=S2254-

38. Revista INFAC volumen $19 n^{\circ} 1$ año 2011 España Adherencia al tratamiento farmacológico en patologías crónicas.

39. Yu ZL, Yeoh LY, Seow YY, Luo XC, Griva K. Evaluation of adherence and depression among patients on peritoneal dialysis. Singapore Med J $2012 \mathrm{Jul} ; 53(7): 474-480$.

40. Vinagre Rea Gema, Arribas Cobo Patricia, Callejo Cano Irene, Martínez Aranda $M^{a}$ Amor, García Estévez Sonia. Bioimpedancia: herramienta habitual en los cuidados de los pacientes de diálisis peritoneal (DP). Rev Soc Esp Enferm Nefrol [revista en la Internet]. 2011 Sep [citado 2015 Sep 21]; 14(3): 155-161. Disponible en: http://scielo.isciii. es/scielo.php?script=sci_arttext\&pid=S1139$13752011000300002 \& \operatorname{lng}=e s$.)

41. Wong FKY, Chow SKY, Chan TMF. Evaluation of a nurse-led disease management programme for chronic kidney disease: a randomized controlled trial. Int J Nurs Stud 2010;47(3):268-278.

42. Lam L, Lee D, Shiu A. The dynamic process of adherence to a renal therapeutic regimen: Perspectives of patients undergoing continuous ambulatory peritoneal dialysis. Int J Nurs Stud, 2014, 51 : 908-916.

43. Téllez T Álvaro. Prevención y adherencia: Dos claves para el enfrentamiento de las enfermedades crónicas. Rev. méd. Chile [Internet]. 2004 Dic [citado 2015 Nov 14]; 132( 12 ): 1453-1455.

44. Heredia Fernández J, Lorente Méndez C, Astorga Pérez F, Chica Arellano J. Factores diferenciadores en la elección de tratamiento sustitutivo renal en nuestra consulta de enfermedad renal crónica avanzada. Enfermería Nefrológica 2013;16:36-37.

45. Pastor J. L, Julián J.C. Claves del proceso de información y elección de modalidad de diálisis en pacientes con insuficiencia renal crónica. Nefrología: Volumen 1.Suplemento 1. 20105: 15-20.

46. Curtis BM, Ravani P, Maberti F, Kennett F, Taylor PA, Djurdjev 0, et al. The short and long term impact of multidisciplinary clinics in addition to standard nephrology care on patient outcomes. Nephrol Dial Transplant. 2005; 20: 147-154.

47. Goffin E, Pirson $Y$, Van Ypersele de Strihou C. Implications of chronic hepatitis B or hepatitis $C$ infection for renal transplant candidates. Nephrol, Dial \& Transplant 1995;10(Suppl. 6):88-92.

48. Gago MC, Martínez S, Sesmero C, Andrés $M M$, Velayo $P$, Hernández $E$, et al. Influencia de la consulta predialisis en los pacientes con enfermedad renal crónica avanzada. Enferm Nefrol. 2007; 1 (32): 76-80.

49. Zamora I, Sanahuja MJ. Enfermedad renal crónica. AEP. 2008; 21: 231-239.

50. Marrón B, Martínez JC, Salgueira M, Barril G, La- mas JM, Martín M, et al. Analysis of patient flow into dialysis: role of education in choice of dialysis modality. Perit Dial Int. 2005; 25 Supl 3: S56-S59.

51. Arnau Vives $M$, Rueda Jarque $M$, Vilplana Molto M, Pernas Pérez C. Proyecto de implantación de un programa de educación para la salud en el proceso asistencial del paciente trasplantado renal. Revista de la Sociedad Española de Enfermería Nefrológica 2005;8(3):7-10.

52. Nygardh A, Wikby K, Malm D, Ahlstrom G. Empowerment in outpatient care for patients with chronic kidney disease - from the family member's perspective. BMC Nurs 2011 Oct 28;10:21-695510-21.

53. Thanamayooran S, Rose C, Hirsch DJ. Effectiveness of a multi- disciplinary kidney disease clinic in achieving treatment guide- line targets. Nephrol Dial Transplant 2005; 20: 2385-93. 